Interfaces

\title{
Entre pastiche pictural et parodie textuelle, Pier Paolo Pasolini twiste avec la Bible
}

\section{Cécile Sorin}

\section{(2) OpenEdition}

1 Journals

Édition électronique

URL : http://journals.openedition.org/interfaces/294

DOI : 10.4000/interfaces.294

ISSN : 2647-6754

Éditeur :

Université de Bourgogne, Université de Paris, College of the Holy Cross

\section{Édition imprimée}

Date de publication : 1 janvier 2016

Pagination : 117-128

ISSN : 1164-6225

\section{Référence électronique}

Cécile Sorin, «Entre pastiche pictural et parodie textuelle, Pier Paolo Pasolini twiste avec la Bible», Interfaces [En ligne], 37 | 2016, mis en ligne le 19 mars 2018, consulté le 07 janvier 2021. URL : http:// journals.openedition.org/interfaces/294; DOI : https://doi.org/10.4000/interfaces.294

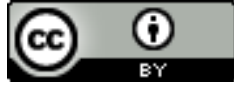

Les contenus de la revue Interfaces sont mis à disposition selon les termes de la Licence Creative Commons Attribution 4.0 International. 


\title{
ENTRE PASTICHE PICTURAL ET PARODIE TEXTUELLE, PIER PAOLO PASOLINI TWISTE AVEC LA BIBLE
}

\author{
Cécile Sorin
}

Confirmant l'adage selon lequel les textes les plus connus sont les plus parodiés, la Bible s'offre effectivement comme la cible par excellence des processus carnavalesques, ce dont témoigne l'ouvrage La Bible Parodiée de Bernard Sarrazin, entièrement consacré aux détournements des principaux épisodes de la Bible.

La littérature comme le cinéma ont ainsi pu en détourner situations et personnages à l'instar de Pier Paolo Pasolini. Toutefois, ce dernier entretient à ce texte une relation mouvante, pouvant aller de la relecture spirituelle et avalisée par l'Église ${ }^{1}$ (L'Évangile selon Saint Matthieu) à la réécriture la plus irrévérencieuse qui soit, La Ricotta ayant d'ailleurs été poursuivie en justice pour outrage.

Ce dernier film, court métrage du poète, raconte l'histoire malheureuse d'un figurant, Stracci, jouant un des larrons crucifiés dans un film sur la vie de Jésus, et qui mourra sur la croix, d'une indigestion, pendant le tournage. Souffre-douleur de la troupe, Stracci, pauvre diable affamé, déploie de nombreuses stratégies pour trouver de la nourriture et n'y tenant plus, dévore une des natures mortes du décor.

Hervé Joubert Laurencin lit dans le titre même du film la métaphore du dédoublement puisque le mot « ricotta » signifierait re-cuite : le récit de la Bible serait recuit, transformé par les opérations qu'il subit tout en étant dédoublé dans la structure même du film. (88) En effet, le film dans le film donne lieu à la reconstitution de tableaux de maîtres ayant la déposition pour thème, version picturale du récit biblique, tandis que la crucifixion de Stracci en offre une version narrativisée et actualisée.

Se mettent ainsi en place des passerelles entre passé et présent, entre image et récit, multipliant les opportunités de jouer des écarts diachroniques, afin d'offrir une lecture irrévérencieuse et contemporaine de la Bible.

1 Ce film a reçu en 1964 le grand prix de l’Office Catholique International du Cinéma. 


\section{Le pastiche pictural}

Le réalisateur du film dans le film, joué par Orson Welles, conçoit l'adaptation filmique du Nouveau Testament sous la forme de reconstitution de tableaux vivants très largement inspirés de chefs d'œuvres de la peinture maniériste.

La Descente de Croix de Rosso Fiorentino est indubitablement reprise avec fidélité pour ce qui est de la composition centrale. Pasolini la flanque de deux serviteurs noirs, très probablement puisés dans l'iconographie de Véronèse et de la peinture vénitienne. (Marchesini 51) De même, La Déposition de Pontormo est enrichie d'une colonne avec un soldat à l'épée, de deux garçons et d'un chien extraits de Vertumne et Pomone de Pontormo ${ }^{2}$. On notera d'emblée la dimension volontairement hétéroclite de ces ajouts, dont les thématiques sortent de l'iconographie religieuse et introduisent des éléments relevant de la peinture profane tels que les garçons et leur chien. Le cœur de chaque tableau est respecté, toutefois sa composition se voit bouleversée dans son format puisque l'on passe de la traditionnelle composition verticale - celle qui symboliquement relie le terrestre au spirituel - à une composition horizontale qui attache fortement les personnages et les objets à la terre. La colonne tronquée, à la verticale brisée, inscrit, dans ce format d'image qui n'a rien de fortuit, les contingences matérielles de la pesanteur. Les ajouts ne se contentent donc pas d'alourdir les descentes de croix de Fiorentino et Pontormo mais bien de transformer sa symbolique, dans un renversement parodique du ciel vers la terre. La descente de croix, moment de réintégration profane du corps par son abaissement symbolique dans le bas du cadre, est traitée sur le mode du gag lorsque l'échafaudage des corps s'écroule. La descente, de glissement devient chute, rappelant de façon irrévérencieuse le primat de l'adage newtonien sur l'irrationalité religieuse.

Le cadre horizontal est également typique des formats d'images cinématographiques. Il confère à l'inversion parodique une dimension réflexive, soutenue par l'omniprésence sonore de l'équipe de tournage hors champ. Ces voix crient des ordres aux comédiens et superposent à l'iconographie religieuse toute la violence de la hiérarchie du tournage. Les tableaux vivants deviennent ainsi indissociables de leur construction matérielle ; ramenés en permanence à la réalité du tournage, ils ne peuvent ouvrir de brèche fictionnelle permettant un développement du récit biblique dans un espacetemps qui lui serait propre et qui aurait pu être celui des tableaux vivants. Le récit biblique se voit donc refuser toute forme d'espace diégétique dans lequel il pourrait se déployer indépendamment des affres de Stracci.

2 Ces emprunts picturaux ont été analysés avec soin par Alberto Marchesini et Xavier Vert. 
La mise en scène du tableau de Fiorentino s'accompagne d'éléments proprement cinématographiques tels que des mouvements de caméra, de nombreux gros plans ou des effets de sonorisation. À la demande du metteur en scène, le tournage est accompagné d'une musique de Scarlatti dont la grâce sérieuse est intempestivement interrompue par les ordres donnés aux figurants. Ceux-ci sont isolés par des gros plans, chaque déplacement du cadre s'arrête sur le jeu exagéré d'un acteur ou sa difficulté à rester sérieux. Malicieux, ces portraits soulignent la résistance des figurants au rôle qu'ils doivent jouer. Bien que les costumes attirent le regard par leurs couleurs éclatantes, bien que le rôle du figurant soit précisément de n'être qu'un objet du décor, on peut dire avec George Didi-Huberman que les gros plans ouvrent dans le tableau vivant des interstices capables de le rendre réellement vivant, c'est-à-dire animé et humanisé. (180) Le plan d'ensemble du tableau est ainsi dénoncé dans sa nature profondément factice, aucun des figurants ne prenant très au sérieux son travail. Loin d'être transfigurés par leur rôle, les figurants, au contraire, apparaissent avant tout comme des salariés.

Le même procédé est mis en œuvre pour filmer la reconstitution de La Déposition de Pontormo et les gros plans sur les figurants se succèdent jusqu'à la désacralisation finale par la chute de cet échafaudage humain.

La bande-son, comme souvent dans le cinéma de Pasolini, fonctionne en contrepoint des images puisqu'aux charmes malicieux des figurants, elle oppose les vitupérations du metteur en scène. Le twist lancé par erreur au début de la prise de vue crée un effet anachronique saisissant avec la reconstitution du tableau qu'il accompagne. Il rappelle la danse saccadée des figurants sur cette musique en début de film, dont le souvenir vient contredire leur l'immobilité raide et forcée dans le tableau vivant. Invitation à la danse, le twist représente également ce qui dans les années soixante était considéré comme du divertissement de masse et oppose ainsi la culture picturale à l'industrie musicale. Ce jeu de contrepoint sonore désacralise la peinture maniériste en lui ôtant de sa solennité, mais il introduit également un rapport de classe, la culture raffinée du metteur en scène rentrant en conflit avec le divertissement populaire des figurants. Un cri du metteur en scène met fin au twist pour le remplacer par de la musique classique démontrant ainsi l'effectivité de la hiérarchie sociale sur le plateau de tournage.

La chute collective de l'échafaudage humain symbolise le retour à la chair, la prédominance du corps sur le spirituel, l'impossible résurrection tandis que la bande-son, par les ordres aboyés aux figurants, révèle la violence de la société de classe.

Un dialogue entre Stracci et le comédien incarnant le Christ précède cette reconstitution et joue sur l'ambiguiité entre ce que sont les personnages et ce qu'ils représentent. Il développe le thème 
de la soumission et des rapports de domination d'une façon suffisamment ambiguë pour pouvoir être interprétée sous un angle religieux ou social.

Stracci se plaint, il a faim et le Christ lui explique que s'il proteste, il ne l'emmènera pas au paradis. Stracci émet un doute sur la capacité du Christ à lui offrir autre chose que ce qu'il vit déjà et conclut, stoïque, que sa vocation est de mourir de faim.

Stracci explicite très clairement qu'il ne croit pas la religion capable de prendre en charge toute forme de justice, notamment sociale, sur terre ou au ciel. Il sous-entend également que la religion est aussi impuissante que d'autres formes de pouvoirs, désignant probablement de façon implicite les politiques italiens. Ces quelques phrases échangées, en attendant la prise de vue suivante, révèlent la conscience qu'ont les comédiens des rapports de classe. Toutefois cette analyse n'est pas porteuse d'indignation mais demeure teintée par la douce résignation de Stracci. Elle exprime la totale passivité des masses, trop habituées à se soumettre pour porter une colère révolutionnaire. Stracci proteste pour lui-même, sans remettre en cause son mode de vie ni le fonctionnement de la société. Son immense bonté et son fatalisme en font une victime née et c'est bien en cela qu'il représente le sous-prolétariat des borgates.

Tourné en noir et blanc, ce dialogue valorise l'irruption vivifiante de la couleur qui accompagne systématiquement les reconstitutions de tableaux. La beauté singulière, portée par l'éclairage contrasté et les couleurs lumineuses, donne à voir autrement la reconstitution du Pontormo dont la finalité ne peut se réduire à l'unique dénonciation des rapports de classe. Elle nous invite à lire le tableau de Pontormo au-delà de l'échafaudage improbable des corps et à aller chercher, nichés dans cet ensemble, des détails d'une grâce exquise, la courbe d'une nuque ou l'éclat d'un sourire. Ce n'est donc plus ici la tension entre l'ascension de l'âme et la chute du corps inanimé qui retient l'attention de Pasolini. Il écarte toute lecture pieuse de la déposition pour y souligner bien au contraire l'élan vital qui anime un à un chaque personnage, comme si le corps inerte et chromatiquement neutre du Christ n'était finalement qu'un prétexte pour révéler ce bouillonnement de chairs lumineuses, l'exubérance des mouvements soulignés par les plis des étoffes, l'expressivité des regards et la vie portée par le rire collectif qui les anime.

Pasolini valorise les regards à la caméra et les épaules dénudées des adolescents. Sa lecture profondément profane et sensuelle du tableau est également une invitation à le redécouvrir.

Pasolini déshabille la peinture religieuse de son contenu biblique et donne ces corps pour ce qu'ils sont : chair, désirs, besoins. Le profane sortirait l'objet sacré d'une sphère isolée pour le ramener à un usage commun. (Agamben) La Ricotta ne procède pas autrement puisqu'elle associe 
la représentation des personnalités sacrées au genre commun, à l'homme dans ses appétits, dans ses fonctions organiques et platement terrestres. Un homme mené par la satisfaction du bas ventre, dont le désir, attisé par amoncellement indécent de nourriture, répond à l'amoncellement tout aussi indécent des corps dans les tableaux vivants, corps érotisés par la pose, les couleurs et les gros plans.

La faim de Stracci peut ainsi incarner les appétits contrariés par la société italienne, creusant les inégalités en même temps que les frustrations. Pasolini dénonce le rôle des rapports de classe autant que de la religion catholique dans la construction de l'enfer du prolétariat romain, déjà amplement décrit dans ses films précédents et son premier roman. (Pasolini Les Ragazzi, Accattone, Mamma Roma)

\section{De l'antimaniérisme à la comédie italienne}

Les deux tableaux de Fiorentino et Pontormo sont considérés sans ambiguïté comme des œuvres maniéristes. De fait, la fascination sensuelle de Pasolini pour ces tableaux vivants ne l'empêche pas de se moquer de l'obsession formelle du maniérisme, qui ne voit dans le sujet religieux que le simple prétexte à des avalanches de corps invraisemblables, des drapés recherchés ou des couleurs somptueuses :

L'ironie, puisqu'elle est ici bel et bien mise en œuvre, tend à exorciser cet « excès de bon goût » qui peut faire prendre - par une surenchère d'exactitude, de raffinement et de formalisme - le zèle pour la charité. En somme, c'est une leçon d'antimaniérisme qui est ici donnée tout autant que de laïcité à l'italienne. (Levergeois 132-133)

Ainsi, la comparaison entre les natures mortes, reconstituées en début et clôture du film, et les tableaux vivants interroge la capacité des peintres à traiter leur sujet avec humanité et spiritualité. On retrouve le même primat de la composition graphique dans ces deux œuvres de nature différentes, comme si les corps ou les aliments pouvaient être traités de la même façon, dans une savante et surprenante accumulation.

Le passage de la peinture de genre à la peinture religieuse tend donc à dénoncer la superficialité qui anime l'obsessionnelle recherche formelle et virtuose des maniéristes. La recherche formelle éclipsant ce qui fait le sujet religieux, ce dernier trouve refuge dans la partie narrative du film, c'est-àdire le récit du tournage. 
Ce déplacement du sujet religieux est manifeste dès les premières minutes du film lorsque Stracci, bien qu'affamé, sacrifie son repas pour l'offrir à sa femme et ses enfants, se faisant ainsi l'écho du partage chrétien, de la symbolique du pain rompu et de l'abnégation. Ce transfert des valeurs chrétiennes sur le personnage de Stracci prendra forme visuellement et deviendra explicite lorsqu'il sera mis en croix pour les besoins de la mise en scène du film dans le film.

Entre le saint invitant de jeunes garçons à une prostitution à peine voilée, le striptease d'une figurante, ou le travestissement de Stracci en blonde pour subtiliser un panier-repas, Pasolini ne se prive pas de construire des doubles parodiques, des inversions carnavalesques jouant habilement sur la dualité inhérente à ces personnages filmiques, partagés entre ce qu'ils doivent incarner — des personnages bibliques - et ce qu'ils sont, des figurants exploités. Cette torsion participe à l'érosion des figures saintes, dont l'image se voit contaminée par cet appel du bas ventre et l'ouverture au réel ainsi suggérée.

Le réalisme du quotidien de Stracci, les plans sur la banlieue de Rome sont autant d'éléments tirés du réel, mais également de la comédie italienne. Ce genre typique du cinéma italien d'aprèsguerre, héritier du néoréalisme, est riche en personnages issus des milieux populaires, aussi loquaces qu'affamés, assumant avec bonhomie leur sort de miséreux. Ils mènent, comme Stracci et les figurants du film, une vie chaotique, faite de débrouillardise et de délinquance, et décrite sous un angle tragicomique avec un réalisme aigu. Les figurants du film sont à la fois " affreux sales et méchants ", ${ }^{3}$ et viennent pervertir toute possibilité d'une représentation pieuse. Ils s'éloignent du tournage déguisés et disséminent ainsi l'iconographie religieuse dans la banlieue romaine, créant ainsi de surprenants effets anachroniques visuels.

\section{Dédoublement narratif}

Lors d'un entretien, Jean-Louis Comolli, critique aux Cahiers du Cinéma, fait la remarque suivante à Pasolini :

Pour en venir à vos propres films, il me semble que c'est précisément l'un des traits de Mamma Roma, par exemple, que de comporter ces deux films : un premier, sorte de documentaire sur les drames de l'existence des «petits » dans la banlieue romaine, un second, en filigrane du premier, où se déroule une sorte de parabole christique... (Bertolucci et Comolli 39)

${ }^{3}$ D'après le titre d'une célèbre comédie italienne d'Ettore Scola : Affreux, Sales et Méchants. 
Or, La Ricotta entretient des liens explicites avec Mamma Roma, réalisé en 1962. Par exemple, le metteur en scène du film dans le film tient à la main l'édition de la novélisation de Mamma Roma. En poursuivant l'analyse de Jean-Louis Comolli, il est possible d'émettre l'hypothèse que La Ricotta, à son tour, repose sur ce dédoublement discursif, mais en l'inversant : l'histoire du Christ devient le lieu où s'exprime toute la grandeur d'âme du sous-prolétariat. Avec une construction narrative éminemment réflexive, Pasolini conte une histoire, celle de Stracci, qui en contient une autre, la crucifixion. Par un effet de contamination et de glissement de valeurs, la vie de Stracci se transforme en celle du Christ tandis que le destin de ce dernier se teinte d'une couleur sociale ; il est bel et bien le symbole des sacrifiés de l'économie de marché.

De nature complexe, la narration du film ne se contente donc pas d'ouvrir un récit dans le récit, mais construit un jeu d'interrelations particulièrement denses entre ces deux histoires. La notion même de parabole, employée par Comolli, rappelle à quel point Pasolini cinéaste a pu user de formes narratives courtes puisqu'il a son actif six courts métrages, des adaptations de contes (Les Mille et Une Nuits) ou de nouvelles enchâssées (Le Décameron). La parabole, forme allégorique typique de la Bible, constitue le type même du récit enchâssé, et invite le lecteur à déployer la symbolique des événements narrés afin d'accéder à son contenu moral.

La Ricotta, par sa forme narrative, détourne la parabole christique et brouille volontairement les pistes d'une éventuelle lecture allégorique par une surcharge signifiante, par un réseau intertextuel complexe mêlant citations musicales ou poétiques, parodies picturales et références cinématographiques.

Aussi il n'est pas étonnant que Pasolini réponde à Comolli, non pas tant sur la possibilité de deux films cohabitant en un seul, mais bien sur la dimension proprement référentielle de cette structure portée par « [...] la technique des citations ». (Bertolucci et Comolli 40), c'est-à-dire la convocation du discours d'autrui enchâssé dans un objet autre.

Le film dans le film fonctionne également sur le principe du récit enchâssé. Cette construction est celle, à un niveau structurel autre, du discours indirect libre, forme linguistique abondement théorisée par Pasolini ${ }^{4}$ et également typique de ses propres modalités narratives et stylistiques puisque Pasolini figure parmi les romanciers analysés dans un des tous premiers textes de linguistique italienne sur le sujet (Herczeg 262-264) : un discours premier en contient un second dont on sait que l'énonciateur du discours premier n'est pas l'auteur, mais qui n'en porte pas moins les marques de cette appropriation.

$4 \quad$ Voir par exemple L'Expérience hérétique. 
Le Nouveau Testament, texte intertextuel s'il en est, apparaît ainsi vu à travers les différents évangiles, à travers leurs multiples interprétations iconographiques, comme un perpétuel processus d'interprétation-création, une chaine de relectures dont La Ricotta ne serait qu'un maillon. Ainsi mise en abyme, La Bible prend à son tour, comme récit, une valeur réflexive. Dépouillée de l'autorité de la lettre, elle n'est plus Le Texte, mais une déclinaison sur un sujet intemporel : la capacité d'abnégation du peuple. Le doublement du Christ en Stracci ne vaut que par la capacité de Stracci à représenter le sous-prolétariat, tout comme le dédoublement narratif ne vaut que pour sa capacité à susciter une mise en abyme des multiples appropriations de La Bible.

La passion du Christ, par ses nombreuses relectures sacrilèges, est une invitation à la duplication infinie. Artaud (L'histoire vraie de Jésus-Christ), Jarry (La Chandelle verte) ou les Monty Python (La Vie de Brian) s'y sont essayés, pour ne citer que les parodies les plus fameuses et irrévérencieuses. L'éloignement ainsi construit à l'égard du référent signifiant, au sens où pouvait l'entendre Baudrillard, creuse de façon abyssale la distance à l'événement historique. Cet écart incommensurable suggère que le simulacre était déjà présent dans le texte initial, autrement dit, qu'il n'y aurait pas pour Pasolini de texte original, mais un simulacre de texte original, dont la relation au référent, est distendue par les multiples versions qui l'ont précédé. La Bible devient ainsi un pur objet d'interprétation et d'inventions.

La profanation ne se contente donc pas de déplacer le sujet, la figure christique, vers un homme du peuple romain, mais procède également par le déplacement du texte biblique, du sacré intouchable vers la sphère du littéraire, de l'imaginaire, de la culture. Rendu à un usage commun, il devient appropriable par chacun et à même de porter des discours multiples, y compris idéologiques.

Et c'est ainsi qu'il faut entendre l'introduction du film : « Quelle que soit l'interprétation qu'on en donnera, l'Histoire de la Passion évoquée par La Ricotta est pour moi la plus grande qui soit. Et les textes qui la racontent les plus sublimes que l'on ait écrits »..$^{5}$ (Pasolini La Ricotta)

Les textes sur la passion sont sublimes, car ils touchent à une forme d'absolu, mais un absolu de nature esthétique et non pas religieuse. Le Nouveau Testament est appréhendé par Pasolini comme une œuvre littéraire dans une relation créative inévitablement profane.

«Ebbene, io voglio qui dichiarare che comunque si prenda La ricotta, la storia della Passione, che indirettamente La ricotta rievoca, è per me la più grande che sia mai accaduta, e i testi che la raccontano i più sublimi che siano mai stati scritti ». 
La Ricotta serait le film de Pasolini le plus proche du carnavalesque au sens où l'entendait Bakhtine :

La Ricotta est, comme d'autres films de Pasolini, constituée de collage et de pastiche de différents langages mis bord à bord : langues du cinéma et de la peinture, l'écriture et l'image, le populaire et le décadent, le film muet et le son. Et ses excès, comme dans le carnaval, en renversant le conventionnel, constituent quelque chose de nouveau, formellement et stylistiquement radical. ${ }^{6}$ (Rohdie 149 )

Le pastiche des tableaux maniéristes associé à la parodie de la bible participe du fonctionnement carnavalesque et subversif du film, tenant autant à la façon dont les voix et discours se mêlent jusqu'à la confusion, qu'à la forme hybride du film. (Rohdie 150)

Alberto Marchesini souligne ce jeu d'inversion typiquement carnavalesque :

Le tableau vivant conjugue de façon exemplaire, dans un véritable oxymoron, les antithèses qui organisent La Ricotta : l' « élevé » et le « bas » (Scarlatti et le twist, la faim d'esthétique du metteur en scène et celle, réelle, de Stracci), l'immobilité et le mouvement (l'aspect statique de Welles et la frénésie de Stracci ), le sacré et le profane, la réalité et la fiction. ${ }^{7}$ (54)

Loin de se réduire à un jeu d'oppositions, ces éléments se complètent et participent d'une dynamique carnavalesque. Ainsi, ce ne sont pas tant différents mondes et valeurs qui s'excluent l'un l'autre par leur mise en relation, mais bien plutôt un parcours qui, à l'instar du fonctionnement du carnaval, destitue pour couronner et se moque pour sacrer selon une logique cyclique de création/destruction immuable.

La Ricotta nous invite ainsi à une lecture carnavalesque, donc cyclique de La Bible : il n’y aurait pas un Christ, mais des christs, dont la vie est faite de misère et de sacrifices. La résurrection devient un événement quotidien. Il ne sert donc à rien de pleurer Stracci car, comme le Christ, il est immortel mais à sa façon puisque d'autres enfants de prolétaires, peut-être les siens, le remplaceront. Voilà pourquoi la reconstitution de La Déposition du Pontormo peut s'achever sur un éclat de rire, ode à la vie et au renouveau.

6 « La Ricotta was, like other films of Pasolini, a collage and pastiche of languages set at the edge of each other: languages of film and painting, writing and image, the popular and decadent, silent film and sound. And its excesses, as in carnival, by overturning the conventional, constituted something formally new and stylistically radical. »

7 «Il tableau vivant conjuga esemplarmente, in un vero e proprio ossimoro, le antitesi su cui si imposta La ricotta: 1' "alto" e il "basso" (scralatti e il twist, la fame estetica del regista e quella reale di stracci), l'immobilità e il movimento (la staticità di Welles e la frenesia di Stracci), il sacro e il profano, la realtà e la finzione.” » 
138

Interfaces 37 (2015-2016)

Plus encore, en considérant la Bible comme une œuvre littéraire, comme un espace imaginaire appropriable par les artistes, Pasolini invite les spectateurs à voir en cette dernière un document intertextuel aux multiples interprétations littéraires ou iconographiques. La Ricotta, quant à elle, ne compte pas moins de deux interprétations explicites du récit biblique auxquelles s'ajoute la charge signifiante des nombreux emprunts. La première, portée par Pasolini lui-même, réactualise la figure du Christ sous les traits de Stracci ; la seconde, portée par le metteur en scène du film dans le film, réplique de façon guindée et prétentieuse la vision exprimée par les tableaux de maître.

Sans extrapoler sur l'influence que la pensée de Pasolini a pu exercer sur l'œuvre d'Agamben, cette dernière affleure dans Profanations, qu'il s'agisse de la réflexion sur Sade ou de la présence de la parodie dans l'espace littéraire italien, à tel point qu'il est possible de voir dans La Ricotta, non pas l'illustration, mais une des sources de l'affirmation suivante : « Du mystère, on ne peut offrir qu'une parodie : toute autre tentative pour l'évoquer tomberait dans le mauvais goût et l'emphase. C'est pourquoi la parodie par excellence est la représentation du mystère moderne : la liturgie de la messe » . (Agamben 45)

Profané, Le Nouveau Testament, de texte sacré est devenu un espace imaginaire. Il peut alors susciter des appropriations alternatives contournant la symbolique du culte pour se tourner vers le partage, non pas de représentations, mais d'une expérience subjective avec L'Évangile selon Saint Matthieu. 


\section{BiBLIOGRAPHIE}

AGAMBEN, Giorgio. Profanations. Paris : Payot \& Rivages, 2005.

ARTAUD, Antonin. «L'Histoire Vraie de Jésus-Christ ». Lignes 6 (février 1989).11-28.

BAKHTINE, Mikhaïl. L'Euvre de François Rabelais et la Culture Populaire au Moyen-Age et Sous la Renaissance. Paris : Gallimard, 1970.

BERTOLUCCI, Bernardo et COMOLLI, Jean-Louis. «Entretien avec Pasolini ». Cahiers du Cinéma hors série. 1981. 35-42.

DIDI-HUBERMAN, Georges. Peuples Exposés, Peuples Figurants - L'œil de l'Histoire 4. Paris : Les Editions de Minuit, 2012.

HERCZEG, Giulio. Lo stile indiretto libero in italiano. Florence : G.C. Sansoni editore, 1963

FIORENTINO, Rosso. La Descente de Croix. Peinture. 1521. Pinacothèque, Voltera.

JARRY, Alfred. La Chandelle Verte : Lumières sur les Choses de ce Temps. Bègles : Le Castor astral, 2006.

JONES, Terry, réal. La Vie de Brian [Life of Brian]. UGC-Fox Distribution. 1979.

JOUBERT-LAURENCIN, Hervé. Pasolini : Portrait du Poète en Cineaste. Paris : Cahiers du Cinéma, 1995.

LEVERGEOIS, Bertrand. Pasolini - L'alphabet du Refus. Paris : Editions du Felin, 2005.

MARCHESINI, Alberto. Citazioni Pittoriche nel Cinema di Pasolini (da Accatone al Decameron). Florence: La Nuova Italia Editrice, 1994.

PASOLINI, Pier, Paolo, Les Ragazzi [Ragazzi di vita]. 1958. 10/18. Paris. 1998 , réal. Accatone. Gaumont. 1961

, réal. Mamma Roma. Carlotta Films. 1962

, réal. La Ricotta. Cineriz. 1963.

, réal. L'Évangile selon Saint Matthieu [Il Vangelo Secondo Matteo]. Rebis. 1964.

, réal. Le Décaméron [Il Decameron]. Produzioni Europee Associati (PEA). 1971.

, réal. Les Mille et Une Nuits [Il Fiore delle Mille e Una Notte]. United Artists Europa. 1974.

, L'Expérience hérétique - Langue et Cinéma. Paris : Payot, 1976.

PONTORMO. La Déposition. Peinture.1527. Eglise Santa Felicità Chapelle Capponi. Florence.

ROHDIE, Sam. The Passion of Pier Paolo Pasolini. London / Bloomington : British Film Institute \& Indiana University Press, 1995.

SARRAZIN, Bernard. La Bible parodiée. Paris : Editions du Cerf, 1993.

SCOLA, Ettore, réal. Affreux, sales et méchants [Brutti, sporchi e cattivi]. Gold Film. 1976.

VERT, Xavier. La Ricotta. Lyon : Aléas, 2011. 